\title{
Efectos adversos del tratamiento del cáncer oral
}

\author{
Silvestre-Donat FJ*, Puente Sandoval A**
}

\section{RESUMEN}

En este estudio se hace una revisión de los efectos adversos más frecuentes por la cirugía de tumores de cabeza y cuello, la radioterapia y la quimioterapia, pues no es infrecuente que el odontólogo general encuentre en su consulta complicaciones como mucositis, xerostomía, necrosis óseas, alteraciones gustativas y otras lesiones que causarán molestias considerables al paciente, disminuyendo su calidad de vida. El papel del odontólogo en el tratamiento multidisciplinario constituye un pilar importante en la prevención, el tratamiento de dichas complicaciones y la disminución de sus secuelas.

Palabras clave: Cáncer, cáncer oral.

\section{SUMMARY}

In this article a revision of most frequent adverse effects of head and neck surgery, radiotherapy and chemotherapy is maid, because frequently general dentists deal with complications at their practises like mucositis, xerostomia, osteonecrosis, taste alterations and other pathologies which will cause disturbances to the patients, affecting their quality of live. The role of the dentist in the multidisciplinary treatment is an important element in the prevention, treatment of the complications and in the reduction of its consequences.

Key words: Cancer, oral cancer.

Fecha de recepción: Diciembre 2007.

Aceptado para publicación: Diciembre 2007.

* Profesor titular de Estomatología. Departamento de Estomatología de la Universidad de Valencia. Jefe de la Unidad de Estomatología del Hospital Universitario Dr. Peset de Valencia.

** Odontólogo de la Unidad de Estomatología del Hospital Universitario Dr. Peset de Valencia..

Silvestre-Donat FJ, Puente Sandoval A. Efectos adversos del tratamiento del cáncer oral. Av. Odontoestomatol 2008; 24 (1): 111-121.

\section{INTRODUCCIÓN}

El tratamiento del cáncer tiene actualmente un enfoque multidisciplinario. Desde que se diagnóstica clínica e histopatológicamente hasta que comienza el tratamiento específico, se implican varios especialistas en un comité oncológico donde se planifi- ca y trata de forma protocolizada la estrategia a seguir (1).

En el cáncer de cabeza y cuello, y más específicamente en el carcinoma de células escamosas, las armas terapéuticas más eficaces pasan por la cirugía y la radioterapia, aunque en determinadas circuns- 
tancias y en otros tipos de cáncer es importante el papel de la quimioterapia. Es importante la prevención y el diagnóstico precoz ya que el tratamiento sería más conservador respecto a la cirugía ablativa evitando extensas resecciones quirúrgicas y evitando tratamientos más agresivos que van a disminuir posteriormente la calidad de vida de estos pacientes. Cuando existe un diagnóstico precoz puede haber una supervivencia a los 5 años de un $80 \%$, en general es de un $55 \%$, mientras que cuando se diagnostica en estadios avanzados no llega al 35\% (2).

A pesar de los avances de los últimos años, el tratamiento de estos procesos patológicos sigue siendo complejo teniendo que utilizar una terapéutica agresiva con hospitalizaciones prolongadas y protocolos de tratamiento que presentan una alta morbilidad. Tanto el tratamiento con cirugía oncológica, como con radioterapia y quimioterapia van a dejar secuelas o efectos adversos $(2,3)$.

\section{LA CIRUGÍA ONCOLÓGICA}

La cirugía oncológica de cabeza y cuello va a producir defectos tras la exéresis de los tejidos afectados y su margen de seguridad. Entre estos defectos podremos encontrar cicatrices y fibrosis, deformidades con afectación en la estética facial tras una maxilectomía o mandibulectomía, defectos por falta de sustancia que crean comunicación entre cavidades como las oronasales u orosinusales y deterioro de las funciones bucales con pérdida de la capacidad defensiva de los tejidos orales.

Estos defectos van a tener que ser reparados mediante la cirugía reconstructiva o mediante prótesis maxilofaciales como los obturadores que tapan y sellan las comunicaciones entre cavidades, o las epítesis faciales que rellenan y maquillan los defectos a nivel de la cara (4).

\section{LA RADIOTERAPIA}

La radioterapia puede administrarse como tratamiento primario tras la cirugía, en combinación con la quimioterapia o como tratamiento paliativo. Las dosis necesarias variaran en función de la localización y tipo de tumor aunque la dosis total en tumores de cabeza y cuello oscilará entre los 50 y los 70 Gy en un periodo entre 5 a 7 semanas (5). Las dosis se fraccionan para dar tiempo a la oxigenación de las células tumorales entre las sesiones y hacerlas más radiosensibles, así como por la diferencia de respuesta de reparación subletal entre el tejido tumoral y los tejidos normales (6). Estas dosis totales se fraccionan en dosis de unos 2 Gy al día, 5 días a la semana (7).

Las fuentes de radiación pueden ser externas e internas. La radioterapia externa da por resultado una área más amplia de irradiación hística tendiendo a presentar una menor dosis de radiación por unidad de área. La radioterapia interna o braquiterapia es capaz de proporcionar una exposición de irradiación más alta en un área más pequeña.

Existe un limite importante en el aumento de las dosis, que lo determina la tolerancia de los tejidos adyacentes normales. Los tejidos con un recambio celular rápido presentarán efectos adversos a la radiación más tempranos que aquellos que tengan un recambio celular lento. Uno de los objetivos del tratamiento radioterápico del cáncer es aumentar la probabilidad de control del tumor y disminuir la probabilidad de complicaciones en los tejidos normales. Para ello se han creado una serie de estrategias como aplicar las dosis con hiperfraccionamiento o fraccionamiento acelerado y el uso de técnicas de planificación computarizada con una intensidad modulada en cada área $(8,9)$.

Los efectos adversos o secundarios a la radioterapia en los tejidos orales pueden ser agudos como las mucositis o la pérdida del sentido del gusto, o crónicos como la hiposialia, las caries dentales, la aparición de trismos o la osteorradionecrosis.

\section{Mucositis}

La mucositis es una reacción inflamatoria de la mucosa orofaríngea por efecto directo de la radiación sobre la mucosa. Se produce por la destrucción de los queratinocitos basales no pudiendo realizar éstos su recambio (10). Es en realidad una atrofia del tejido escamoso epitelial en ausencia de daño vascular 
y con un infiltrado inflamatorio en el área basal. En un alto porcentaje de casos hay un exudado de fibrina hacia la superficie, dando lugar a pseudomembranas (11).

Muchos son los factores que pueden contribuir al desarrollo de la mucositis como son el incremento de factores mediadores de la inflamación, como el incremento del factor activador de las plaquetas que aumenta en saliva, la adhesión de leucocitos a selectina $\mathrm{E}$ o la disminución del nivel de factor de crecimiento epidérmico. Así mismo, se ha observado un detrimento en los factores de defensa o protección de la mucosa como la integridad de su estructura, la disminución de secreción salival o la IgA salival, la disminución del recambio celular y la alteración en la flora habitual (12). Parece ser que la mucositis puede agravarse por la aparición de microorganismos gran negativos o de micosis. Hay factores sistémicos que de igual forma tienen influencia en la aparición de la mucositis como la edad avanzada, la cirugía previa y la existencia de enfermedades subyacentes (13).

Desde dosis pequeñas de radiación, en torno a 20 Gy, pueden comenzar a manifestarse pequeñas lesiones, sin embargo, cuando estas dosis aumentan dichas lesiones se manifiestan más marcadamente en la clínica. En principio puede aparecer un eritema sobre la mucosa seguido de ulceraciones que permiten la alimentación por vía oral. En un grado más avanzado estas ulceraciones van creciendo y llegan a permitir sólo la alimentación blanda o líquida. La extensión de las lesiones a un grado máximo no permitirá la alimentación por vía oral (14).

Clínicamente se producen molestias locales iniciales seguidas por dificultad para beber, comer, tragar o hablar. Suelen comenzar a la semana de tratamiento radioterápico y duran hasta 2-3 semanas después de finalizadas las dosis. Hay que valorar la presencia de infecciones oportunistas que puedan complicar su evolución. El diagnóstico es clínico (13).

En la literatura sobre el tema, el manejo de la mucositis esta basado más en la experiencia clínica que en ensayos clínicos controlados. No hay fármacos que puedan prevenir la aparición de mucositis, los que hay se limitan a reducir su severidad, a controlar el dolor y a eliminar los microorganismos involucrados.

El tratamiento será preventivo y paliativo de las complicaciones. Se basará en evitar los factores irritativos sobre la mucosa, mantener una buena higiene y mantener la humedad sobre la mucosa, aliviando el dolor y la inflamación, así como prevenir y tratar las infecciones orales cuando se presenten.

Se evitarán alcohol, tabaco, especias y comidas muy calientes. Las prótesis removibles se deben haber quitado durante la radioterapia (15). Para la protección de la mucosa se pueden utilizar geles protectores como sucralfato. Los enjuagues o geles con lidocaína al $2 \%$ en solución acuosa pueden beneficiar aliviando el dolor, así como los enjuagues con antisépticos tipo clorhexidina al $0,12 \%$. Algunos autores han evidenciado cierta mejoría en la evolución de la mucositis con el uso de ciertos antibióticos para combatir la sobreinfección por gran negativos o las micosis como la polimixina, la tobramicina o la anfotericina B (16). Igualmente se ha visto mejor evolución al administrar ciertos factores de crecimiento como el factor estimulante de colonias de granulocitosmacrófagos (FEC G-M) y factor de crecimiento epitelial (17).

\section{Alteraciones de la gustación}

La mayoría de los pacientes experimentan de forma temprana una perdida parcial o completa del sentido del gusto disminuyendo exponencialmente desde una dosis total acumulada de 30 Gy (3 semanas) (13). Los sabores que parecen tener un mayor perdida gustativa son el amargo y el ácido frente a salado y dulce. Esta pérdida del sentido del gusto esta producida por la afectación de la radiación sobre las células de los corpúsculos gustativos de las papilas linguales pero también se refuerza en el momento en que comienza la hiposialia con pérdidas discretas de tasas de flujo salival (2). Los botones gustativos que se encuentran principalmente en las papilas fungiformes y circunvaladas son muy sensibles a la radiación y suelen estar afectados porque la lengua casi siempre suele estar incluida en el campo irradiado. Estas células de los botones gustativos suelen regenerarse en unos cuatro meses aunque el grado 
de afectación de un paciente a otro varia ampliamente.

Sin embargo, estas alteraciones casi siempre son transitorias y el sentido del gusto vuelve a aparecer gradualmente recuperando niveles anteriores aproximadamente hasta un año después de finalizada la radioterapia. La recuperación de este sentido por termino medio suele estar entre los 60 y los 120 días después de finalizada la última dosis. Por lo tanto, no es necesario un tratamiento específico para esta alteración si bien se deben variar algo los patrones de alimentación y realizar un consejo dietético con comidas con apariencia agradable debido a que se reduce el apetito en este periodo. Hay autores que han aconsejado suplementos de zinc, para ayudar a recuperar el sentido del gusto, con dosis de $100 \mathrm{mg}$ al día $(18,19)$.

\section{Alteraciones de la secreción salival}

La xerostomía en los pacientes irradiados por cáncer en cabeza y cuello se produce por la pérdida de tasas de flujo salival (hiposialia) tras la inflamación producida en las glándulas salivales incluidas en el campo irradiado (20). Las glándulas salivales son sensibles a la radioterapia y responden a dosis bajas de radiación aunque sus efectos a esas dosis son reversibles (21). Su origen es por compromiso vascular con afectación de los pequeños vasos que rodean las unidades funcionales salivales. Se produce una atrofia y necrosis de las células acinares y ductales con cambios en el tejido conectivo. La proporción de pérdida de flujo estará en relación a la cantidad de tejido salival incluido en el campo afectado y de la cantidad de dosis suministrada. A dosis acumulativas superiores a 70 Gy pueden ser irreversibles con degeneración y fibrosis del tejido glandular.

Se suelen afectar más las glándulas serosas que las mucosas volviéndose la saliva viscosa (22). Hay como cuatro fases en la pérdida de la función glandular, en los primeros 10 días hay una perdida de flujo con secreción de amilasa, hasta los 60 días va disminuyendo la secreción de amilasa con progresiva pérdida de células acinares luego se entra en una fase que no cambian los parámetros anteriores. Finalmente hay un deterioro del funcionalismo salival pero comienza la recuperación del tejido acinar entre los 120 a 240 días.

Cuando disminuye el flujo salival se produce una reducción en el ph y la capacidad tampón, una reducción en los niveles de electrolitos, cambios en los sistemas antibacterianos y una reducción en la secreción de bicarbonato. Así mismo, se producen cambios en la flora bucal sobre todo a los tres meses postradioterapia con aumentos en las colonias de streptococus mutans, lactobacillus y cándidas.

Clínicamente hay cierta dificultad para desarrollar las funciones bucales pues disminuye la humedad y lubrificación. Hay cierto disconfort nocturno y dificultad para la retención de prótesis removibles, los labios están secos y con cierto grado de descamación y la lengua tiene un aspecto de la mucosa seca y de aspecto fisurada o cuarteada. Puede haber una mayor vulnerabilidad a la aparición de infecciones orales del tipo de las candidiasis (23).

La prevención de estas alteraciones se debe de conseguir mediante un plan de tratamiento radioterápico meticuloso, evitando la radiación de las glándulas salivales y valorando el parénquima salival residual. Si existe posibilidad de estimular las glándulas salivales haremos uso de los sialogogos como la anetol tritiona, la cevimilina o la pilocarpina (24). Una parte de los efectos beneficiosos de la estimulación de la secreción es que actúan sobre las glándulas salivales menores palatinas y de alguna manera el aumento de lubrificación evita la irritación sobre esta zona de la mucosa.

Por otro lado, están los sustitutos de la saliva o saliva artificial que suele estar compuesta de una solución acuosa con sales minerales y otro tipo de sustancias protectoras o lubrificantes como la glicerina, la mucina o la carboximetilcelulosa (25).

Dependiendo del grado de hiposialia se pueden utilizar unos productos u otros. En la hiposialia de baja intensidad se darán estimulantes gustativos o farmacológicos. En la de tipo moderado se darán estimulantes salivales farmacológicos y sustitutivos salivales por la noche. En los casos más graves se prescribirán sustitutivos salivales por el día y la noche. 


\section{Caries dentales}

Las caries dentales que aparece en pacientes tras la radioterapia son agresivas y persistentes y se muestran en individuos que nunca habían mostrado predisposición a estas lesiones. Su aparición esta relacionada con varios factores, aunque sin duda es la hiposialia la que mayor influencia tiene. La saliva tiende a disminuir en cantidad, su ph, los electrolitos y la inmunoproteínas. Así mismo, puede influir en su aparición la falta o disminución de los hábitos higiénicos por las molestias que padecen, la sustitución de la dieta habitual por otra más blanda, no detergente y rica en hidratos de carbono y por la aparición de una microbiota oral con características más acidogénicas y cariogénicas (26).

Las lesiones suelen aparecer hacia los tres meses de finalizadas las dosis de radioterapia, son lesiones irreversibles y extensas que afectan a las áreas cervicales dentales, tanto vestibulares como palatinas o linguales, pero también pueden darse en zonas poco habituales como los bordes incisales (27).

Los pacientes presentarán hipersensibilidad a la temperatura y a los alimentos dulces, raramente producen pulpitis aunque pueden llegar a destruir la base de la corona con fracturas a ese nivel.

La prevención de estas lesiones debe comenzar con la motivación del paciente y los consejos dietéticos antes de comenzar la radioterapia. Se debe seguir una dieta suave y equilibrada con suplementos vitamínicos y proteicos. Evitar los alimentos cariogénicos. Se deben restaurar los dientes afectados mediante obturaciones estéticas y siguiendo una higiene correcta con cepillado dental después de las comidas y aplicaciones tópicas de fluoruro en forma de gel en cubetas y usando pasta dentífrica de alto contenido en flúor. De igual forma se pueden hacer aplicaciones de barniz de clorhexidina de forma trimestral (28).

\section{Trismos}

Es la disminución de la apertura bucal que se puede producir tras 3-6 meses postradiación producida por la fibrosis de los músculos masticatorios y de la ATM. En algunas ocasiones puede producirse una limita- ción de la apertura tras lesiones como una fractura mandibular como evolución de osteorradionecrosis. El tratamiento se basará en la administración de relajantes musculares y fisioterapia oral. Para poder seguir la evolución se debería medir la distancia interincisal máxima antes de comenzar la radioterapia para poder comprobar si se pierde apertura y si se va recuperando tras el tratamiento (28).

\section{Osteorradionecrosis}

La lesión más seria tras la radioterapia es la osteorradionecrosis (ORN). Se trata de un área de hueso expuesto, al menos 6 semanas, en un campo irradiado, doloroso y con frecuencia con infecciones repetitivas. Puede evolucionar clínicamente hacia fístula cutánea o mucosa, secuestros óseos o fractura mandibular. La incidencia en mandíbula según autores estaría entre el 5 y el $15 \%$, en maxilares sería algo más baja $(29,30)$.

La causa de esta lesión la encontraríamos en la afectación por la radioterapia de la vascularización ósea y de los sistemas reparativos. La patogenia de esta lesión se basa en la conjunción de factores como la radiación, el trauma y la infección posterior del hueso. El trauma severo actuaría como el portal de entrada de los microorganismos al hueso. Actualmente, la lesión es considerada como un proceso infeccioso a través del hueso que no actuaría de barrera debido a la vascularización comprometida y a una capacidad reparativa mínima. El trauma puede producirse por muchas situaciones como las irritaciones por prótesis dentales, los bordes cortantes de los dientes o por una manipulación clínica como una extracción dental. Aunque existe un 35\% de los casos que se consideran espontáneos (31).

Entre los factores que incrementarían su aparición están, la edad avanzada, las dosis totales superiores a 65 Gy, la inclusión de la mandíbula en el campo irradiado, el Hiperfraccionamiento de las dosis y la combinación entre la radioterapia externa y braquiterapia (32).

El diagnóstico se basará en los antecedentes y en la aparición de signos clínicos. Aunque la clínica puede pasar desapercibida al principio cuando la lesión es 
muy pequeña o puede manifestarse por la aparición de fístulas cutáneas u orales, celulitis y supuración, secuestros óseos o fracturas patológicas. También se manifiesta con olor fétido, sangrado o dolor intenso en la zona.

Para prevenir su aparición se debe mejorar el estado bucodentario antes del tratamiento con radioterapia, dejando suficiente tiempo de cicatrización tras las extracciones dentales o la cirugía oral (al menos 15-20 días) (33).

El tratamiento inicial sería el más interesante con limpieza y exéresis del hueso necrótico afectado bajo cobertura antibiótica. Algunos autores preconizan el uso del oxígeno hiperbárico durante este periodo. Cuando las lesiones son más avanzadas se hace una resección en el área afectada (mandibulectomía) hasta márgenes con hueso de aspecto vascularizado.

En su evolución, las lesiones pueden ser de tipo crónico persistente o bien progresar de forma activa con fractura patológica (34).

\section{LA QUIMIOTERAPIA}

El papel de la quimioterapia en el tratamiento del cáncer de cabeza y cuello ha sido controvertido y limitado, si bien hasta hace pocos años, por presentar su efectividad ante tumores con un importante factor de crecimiento y una elevada tendencia a la diseminación a distancia, propiedades nada frecuentes en los tumores de tipo epidermoide, siendo estos los más prevalentes en cabeza y cuello (35).

Actualmente se emplea de forma combinada con el tratamiento locorregional (cirugía y radioterapia), de forma secuencial, aumentando los resultados de éxito o incluso como tratamiento paliativo en tumores recurrentes o bien en metástasis.

Así mismo en la pauta del tratamiento quimioterápico, basándose en los estudios más recientes, prima el empleo de combinaciones de agentes (poliquimioterapia) frente al tratamiento único (monoquimioterapia), buscando un efecto sinérgico de los fármacos a la vez que la disminución de los efectos adversos.
El papel de la quimioterapia en el tratamiento del cáncer de cabeza y cuello presenta los objetivos de disminuir la metástasis a distancia y aumentar el control local. Este último factor es importante pues un elevado porcentaje de estos causan la muerte por mecanismo locorregional $(35,36)$.

Los fármacos antineoplásicos se clasifican según el tipo de células sobre las que actúan. Así tenemos los cicloindependientes que actúan sobre células en división y reposo. Son específicos de fase, es decir, actúan sobre células en una determinada fase del ciclo celular. Y los ciclodependientes: actúan sobre células en diversas fases del ciclo celular (37).

Así mismo, los fármacos que actúan sobre las células en división, se pueden clasificar según su mecanismo de acción en $(35,37)$ :

- Alquilantes: inhiben la división celular formando enlaces cruzados entre cadenas de DNA impidiendo su replicación.

- Antimetabolitos: inhiben la división celular actuando sobre los enzimas imprescindibles para la síntesis de bases púricas y pirimidínicas.

- Inhibidores de la mitosis: inhiben la división celular actuando sobre los microtúbulos del núcleo, impidiendo su división.

Entre los fármacos antineoplásicos usados en el tratamiento del cáncer de cabeza y cuello tenemos el metrotexate, la bleomicina, el cisplatino, el carboplatino, el 5-fluoruracilo, la gencitabina, la ciclofosfamida, la doxorubicina, la vincristina y la vinblastina (35).

Los fármacos antineoplásicos actúan sobre diversas fases de la cinética celular, bien sobre una o varias fases de la división celular o bien sobre células en reposo, controlando el crecimiento o replicación y/o destruyendo células cancerosas; pero debido a su falta de selectividad, también actúan sobre células normales con una elevada tasa de replicación, causando diversos efectos secundarios que variarán en función de la dosis, ciclos y vías de administración, y que afectarán principalmente a los sistemas digestivo, hematológico, renal, neurológico y dermatológico (37).

Las complicaciones orales relacionadas con la quimioterapia son debidas a múltiples factores, desta- 
cando la lesión letal y subletal de los tejidos orales, la deficiencia inmunitaria y la interferencia con el proceso normal de curación; derivados del daño directo de los tejidos orales secundarios a la quimioterapia y del daño indirecto debido a toxicidad regional/sistémica. Estas complicaciones pueden ser agudas, desarrollándose durante la terapia, o bien crónicas $(37,38)$.

\section{Mucositis oral}

La mucositis oral se define como una reacción inflamatoria a los fármacos quimioterápicos, que se manifiesta como eritema o ulceraciones orales y que puede verse exacerbada por factores locales. Se presenta en un $40 \%$ de los pacientes en tratamiento, requiriendo un $50 \%$ de éstos intervención médica, incluyendo modificación oncoterápica, por la severidad de éstas. Su inicio acontece unos 7 o 10 días del inicio de la quimioterapia, viéndose la mucosa labial y bucal, la lengua, el suelo de la boca y el paladar blando más afectados que los tejidos fijos más queratinizados, como el paladar duro y las encías, lo que puede deberse a una mayor tasa de renovación de las células epiteliales. Es difícil predecir si un paciente desarrollará mucositis basándose en los fármacos, si bien se asocian con más frecuencia con Metrotaxato, Doxorubuicina, 5-Fluorouracilo, Bleomicina y Cisplatino (39).

El manejo de la mucositis oral por medio de métodos tópicos comprende la limpieza atraumática de la mucosa oral mediante enjuagues suaves con solución salina más bicarbonato sódico, el mantenimiento de la lubricación de labios y tejidos orales con fármacos que revisten la mucosa tipo Amphojel, Kaopectate, películas de cianoacrilato, y el alivio del dolor e inflamación con anestésicos tópicos (Lidocaína, Benzocaína, Defenhidramina...) o analgésicos.

La Palifermina, o factor de crecimiento 1 del queratinocito, ha sido aprobado recientemente por la FDA para disminuir la prevalencia y duración de la mucositis oral severa $(40,41)$.

Diversos autores preconizan el uso de la crioterapia, empezando 5 minutos antes y durante 40 minutos, para la prevención de la mucositis oral en fármacos como el 5-Fluorouracilo, cuya semidesintegración es de 5 a 20 minutos; de este modo se reduce la transferencia vascular de éstos fármacos y tóxicos al epitelio oral que se está replicando (40).

\section{Infecciones}

La alteración de las funciones múltiples de la mucosa oral como barrera protectora, la disfunción de las glándulas salivales y la inmunosupresión asociadas a la quimioterapia, pueden desembocar en sobreinfecciones de la mucositis oral, llegando incluso en neutropenias profundas a diseminarse sistémicamente. Periodonto y afectaciones periapicales también pueden infectarse durante la mielosupresión resultante, por lo que será importante el tratamiento odontológico preventivo previo. Los pacientes con enfermedad periodontal crónica pueden desarrollar enfermedades agudas, y sus consecuentes secuelas sistémicas, sin signos inflamatorios por la mielosupresión. Asimismo, deben evaluarse las prótesis removibles y ajustarlas antes del inicio de la quimioterapia, para evitar los traumas sobre la mucosa oral y invasión microbiana $(35,42)$.

La candidiasis es causada por el sobrecrecimiento de Candida Albicans, es debido a los factores mencionados anteriormente, así como por la antibioticoterapia pautada durante la neutropenia prolongada, que altera la flora oral creando una ambiente favorable. En estos casos se ha observado que el tratamiento con Nistatina es incapaz de controlar la infección; se opta por la familia de antifúngicos azoles, como el Fluconazol, que según los últimos estudios presentan los mejores resultados. En caso de riesgo de diseminación sistémica el tratamiento de elección será la Anfotericina B (41).

No son tampoco infrecuentes las infecciones por el grupo del herpes virus (VHS, VVZ, VEB, CMV), bien como resultado de una reactivación de un virus latente o como una nueva infección. Al igual que con otras infecciones, el riesgo de diseminación sistémica, morbilidad y mortalidad aumentan con el grado de inmunosupresión $(39,41)$.

Las lesiones orales por VHS pueden variar desde herpes labial a estomatitis grave. Éstas surgen si- 
multáneamente al tratamiento quimioterápico, siendo la terapia tópica insuficiente, motivo por el cual se opta por el Aciclovir, Valciclovir vía oral.

Las lesiones orofaciales por VVZ se observan varias semanas después de la interrupción del tratamiento, con un periodo de riesgo elevado de 3 a 12 meses aproximadamente. El tratamiento de elección son: Aciclovir, Valciclovir y Fanciclovir $(39,41)$.

Las infecciones por virus no herpéticos se producen en pacientes con una inmunosupresión prolongada, siendo los agentes más frecuentes adenovirus y VPH, presentando lesiones orales de aspecto verrugoides e hiperqueratósicas. La cirugía láser y la crioterapia son efectivas en el tratamiento de éstas $(35,41)$.

\section{Tendencia al sangrado}

Las hemorragias pueden ocurrir por la trombocitopenia o la coagulopatía inducidas por la quimioterapia, pudiendo observarse sangrado espontáneo cuando las plaquetas son $<30.000 \mathrm{Ud} / \mu$ l en presencia de gingivitis o periodontitis preexistente. Incluso la función normal o la higiene oral habitual pueden provocar sangrado en trombocitopenias graves, por lo que habrá que avisar al paciente y a su familia. Por otro lado, no se recomienda la interrupción de la higiene oral, pues aumentará el riesgo de infección oral y sistémica por el acumulo de placa bacteriana y, asimismo, más sangrado (38).

Las úlceras presentes en los tejidos blandos podrán producir sangrados severos, dependiendo de las lesiones.

Vasoconstrictores como la Epinefrina tópica, protectores tisulares mucoadherentes tipo Cianoacrilatos que sellan sitios sangrantes y protegen los coágulos formados, y por último fármacos favorecedores de la coagulación como trombina tópica o colágeno hemostático que organicen y estabilicen los coágulos, constituyen el tratamiento de elección ante las hemorragias (40).

La realización de enjuagues con Peróxido de Hidrógeno rebajado al 3\% puede ayudar a limpiar las heridas y eliminar los restos de sangre, pero debe tener- se cuidado de no perturbar los coágulos, pudiendo provocar nuevas hemorragias (41).

\section{Neurotoxicidad}

Ciertos quimioterápicos como la vincristina y vinblastina pueden causar neurotoxicidad directa, apareciendo dolores mandibulares profundos y palpitantes. Asimismo, el dolor pulpar podrá verse simulado, por lo que será importante la exploración oral completa para descartarse procesos pulpares agudos o subagudos, en cuyo caso, la labor del odontólogo debe encaminarse al apoyo psicológico del paciente y al control del dolor. Los síntomas suelen remitir una semana después del cese del tratamiento.

Ocasionalmente pueden apreciarse periodos de hipersensibilidad dental varias semanas o meses tras la finalización de la quimioterapia; en este caso la aplicación tópica de flúor o desensibilizantes limitarán la incomodidad $(35,42)$.

\section{Disgeusia}

La disgeusia es un síntoma importante en los pacientes que reciben quimioterapia, pues a parte de la afectación por neurotoxicidad directa sobre las células gustativas, se ve potenciada por otros factores como la xerostomía, las infecciones, y el propio condicionamiento psicológico del paciente. Durante la quimioterapia se puede sentir un sabor desagradable secundaria a la difusión del fármaco en la cavidad oral; la disgeusia, per se, se inicia a las pocas semanas de haberse finalizado el tratamiento citotóxico, siendo reversible, por lo general, a las pocas semanas $(35,42)$.

\section{Hiposialia}

Ciertos tratamientos quimioterápicos causan una disminución clínicamente significativa del flujo salival, pues ésta afecta al parénquima glandular; si bien es cierto que la aparición de xerostomía por quimioterapia es menos frecuente que en el caso de la inducida por radioterapia, siendo además transitoria. 
Se ha observado una alteración de los constituyentes de la saliva con un aumento en los niveles de peroxidasa y amilasa, una disminución del volumen total de IgA e IgG secretadas y la presencia del propio quimioterápico, factores éstos asociados que parece que pueden favorecer, según los pocos estudios realizados al respecto, la aparición de mucositis (43).

\section{Osteonecrosis}

La osteonecrosis maxilar asociada al tratamiento quimioterápico del cáncer fue descrita por primera vez en 2003, desde entonces cientos de casos se han reportado de esta complicación. Se asocia con el uso por vía intravenosa de fármacos de la familia de los bifosfonatos, presentándose con mayor frecuencia en pacientes bajo tratamiento con ácido zoledrónico y pamidronato durante un periodo superior a 36 meses, con un porcentaje de aparición, según recientes estudios, de $10 \%$ y $4 \%$ respectivamente (44).

Estos fármacos presentan acción inhibidora de la reabsorción osteoclástica, así como un marcado efecto antiangiogénico; por lo que alteran el proceso normal de reparación ósea ante las microfracturas que se producen a diario en los huesos sometidos a carga y se favorece la formación osteoblástica de un nuevo hueso, con un aporte sanguíneo empobrecido, acumulado sobre hueso no vital, dándose así un ambiente ideal para el desarrollo de la osteonecrosis (44).

La típica presentación clínica es un área de hueso no vital expuesto que puede presentar infección secundaria y el tejido blando circundante suele estar inflamado y ser sensible a la palpación, dificultando la higiene oral. La superficie de este hueso necrótico presenta microfracturas de bordes afilados que pueden traumatizar tejidos blandos, como lengua y mejillas, llegando a causar úlceras y dolor continuo (45).

La necrosis ósea puede aparecer espontáneamente, o más frecuentemente ante un procedimiento dentario invasivo. Se observa una mayor incidencia en el maxilar superior por presentar éste mayor vascularización y, por ello, haber recibido un mayor aporte del fármaco durante el tratamiento quimioterápico.
El tratamiento conservador debe consistir en evitar la progresión de la necrosis, pues actualmente no se conoce ningún tratamiento efectivo. No se han observado resultados significativos en el tratamiento con oxígeno hiperbárico con y sin tratamiento antibiótico agresivo. Por otro lado, la exéresis del secuestro óseo, tratamiento habitual hasta la fecha, suele conllevar el aumento del tamaño del defecto óseo $(45,46)$.

\section{BIBLIOGRAFÍA}

1. Ord RA, Blanchaert RH. Oral Cancer: The dentist's role in diagnosis, management, rehabilitation and prevention. Chicago: Quintessence Publishing Co 2000. p. 81-109.

2. González-Moles MA, García-Asensio J. Efectos adversos del tratamiento del cáncer oral. En: González-Moles MA (ed). Precáncer y cáncer oral. Madrid: Ed Avances Medico-Dentales S.L. 2001. p. 189-218.

3. Werning JW. Oral Cancer. Diagnosis, management and rehabilitation. New York: Thieme Medical Publishers 2007. p. 209-36.

4. Taylor TD. Clinical maxillofacial prosthetics. Chicago: Quintessence Publishing Co 2000. p. 85-143.

5. Vissink A, Jansma J, Spijkervet FKL, Burlage FR, Coppes RP. Oral sequelae of head and neck radiotherapy. Crit Rev Oral Biol Med 2003;14: 199-212.

6. Cox JD. Dose response for local control with hyperfractionated radiation therapy in advanced carcinoma of the upper aerodigestive tracts. Int J Radiat Oncol Biol Phys 1990;18:515-21.

7. Conger AD. Loss and recovery of taste acuity in patients irradiated in the oral cavity. Radiat Res 1973;53:338-47.

8. Rusell NS. Individual variation in normal tissue reactions to radiotherapy: correlations with radiobiological parameters (thesis). Amsterdam: Free University of Amsterdam 2000. 
9. Wu Q, Manning M, Schmidt-Ullrich R, Mohan R. The potential for sparing of parotids and escalation of biologically effective dose with intensity-modulated radiation treatments of head and neck cancers: a treatment design study. Int J Radiat Oncol Biol Phys 2000;46:195205.

10. Scully C, Epstein JB. Oral health care for the cancer patient. Oral Oncol Eur J Cancer 1996;32:281-92.

11. Silverman S Jr. Diagnosis and management of oral mucositis. J Support Oncol 2007;5:13-21.

12. Handschel J, Prott FJ, Sunderkötter C, Metze D, Meyer U, Joos U. Irradiation induces increase of adhesion molecules and accumulation of â2integrin-expresing cell in humans. Int $\mathrm{J}$ Radiat Oncol Biol Phys 1999;45:475-81.

13. Pico JL, Avila-Garavito A, Naccache P. Mucositis: Its ocurrence, consecuences and treatment in the oncology setting. Oncologist 1998; 3:446-51.

14. Sonis ST. Mucositis as a biological process: a new hypothesis for the development of chemotherapy induced stomatotoxicity. Oral Oncol 1998;34:34-43.

15. Zimmermann JS, Niehoff P, Wilhelm R, Schneider R, Kovàcs G, Kimmig B. Prophylaxe und therapie akuter strahlenfolgen an haut und sleimhaut. Teil II: Empfehlungen der literatur. Strahlenther Onkol 1998;174:187-92.

16. Sonis ST. Pathobiology of oral mucositis: novel insights and opportunities. J Support Oncol 2007;5:3-11.

17. Dörr W, Noack R, Spekl K, Farrell CL. Modification of oral mucositis by keratinocyte growth factor: single radiation exposure. Int $\mathrm{J}$ Radiat Biol 2001;77:341-7.

18. Vissink A, Burlage FR, Spijkervet FKL, Jansma J, Coppes RP. Prevention and treatment of the consequences of head and neck radiotherapy. Crit Rev Oral Biol Med 2003;14:213-25.
19. Matsuo R. Role of saliva in the maintenance of taste sensitivity. Crit Rev Oral Biol Med 2000; 11:216-29.

20. Eisbruch A, Ship JA, Kim HM, Ten Haken RK. Partial radiation of the parotid gland. Sem Radiat Oncol 2001;11:234-9.

21. Nagler RM. The enigmatic mechanism of irradiation-induced damage to the major salivary glands. Oral Dis 2002;8:141-6.

22. Coppes RP, Vissink A, Konings AWT. Comparison of radiosensitivity of rat parotid and submandibular glands after different radiation schedules. Radiother Oncol 2002;63:321-8.

23. Grötz KA, Genitsariotis S, Vehling D, Al-Nawas B. Long-term oral Candida colonization, mucositis and salivary function after head and neck radiotherapy. Support Care Cancer 2003;11: 717-21.

24. Silvestre-Donat FJ, Miralles-Jordá L, MartinezMihi V. Tratamiento de la boca seca : puesta al día. Med Oral 2004;9:273-9.

25. Wynn RL, Meiller TF. Artificial saliva products and drugs to treat xerostomia. Gen Dent 2000;48: 630-6.

26. Johansson G, Andersson G, Attstom R, Edwardsson S. Oral mucous membrane flora in patients using saliva substitutes. Gerodontology 2000;17:87-90.

27. Silvestre FJ, Bagán JV, Del Olmo JA, Gimeno V. État bucco-dentaire chez le patient accoutumé aux drogues: étude de 66 cas. Actualités OdontoEstomatologiques 1990;170:299-306.

28. Silvestre FJ, Plaza A, Serrano C. Prevención y tratamiento de las complicaciones derivadas de la radioterapia en los pacientes con tumores de cabeza y cuello. Med Oral 1998; 3:136-47.

29. Marx RE. Osteoradionecrosis. A new concept of its pathophysiology. J Oral Maxillofac Surg 1983;41:283-8. 
30. Constantino PD, Friedmsn CD, Steinberg MJ. Irradiated bone and its management. Otolaryngol Clin North Am 1995;28:1021-38.

31. Marx RE, Johnson RP. Studies in the radiobiology of osteoradionecrosis and their clinical significance. Oral Surg Oral Med Oral Pathol 1987;64:379-90.

32. Rubira CM, Devides NJ, Ubeda LT, Bortolucci AG Jr, Lauris JR, Rubira-Bullent IR, Damante JH. Evaluation of some oral postradiotherapy sequelae in patients treated for head and neck tumors. Braz Oral Res 2007;21:272-7.

33. Mendenhall WM. Mandibular osteoradionecrosis. $\mathrm{J}$ Clin Oncol 2004; 22:4867-8.

34. Schwartz HC, Kagan AR. Osteoradionecrosis of the mandible. Scientific basis for clinical staging. Am J Clin Oncol (CCT) 2002; 25:168-71.

35. Yogi V, Singh OP. Induction followed with concurrent chemo radiotherapy in advanced head E neck cancer. J Cancer Res Ther 2005; 1:198203.

36. Nguyen NP, Moltz CC, Frank C, Vos P, Smith HJ, Karlsson U, Dutta S, Midyett FA, Barloon J, Sallah $\mathrm{S}$. Dysphagia following chemoradiation for locally advanced head and neck cancer. Ann Oncol 2004; 15:383-8.

37. Borowski B. Les soins bucco-dentaires du malade cancereux. Paris :Masson 1986. p.60-5.

38. Villar-Rodríguez A. Cáncer de cabeza y cuello. Monografías Clínicas en Oncología 3. Barcelona: Ed. Doyma S.A. 1989. p.29-34.
39. Scully C, Epstein JB. Oral mucositis: a challenging complication of radiotherapy, chemoterapy and radiochemotherapy. Part 2: diagnosis and management of mucositis. Head Neck 2004;26:77-84.

40. Lalla V, Schubert M. Anti-inflamatory agents in the management of alimentary mucositis. Support Care Cancer 2006;14:558-65.

41. Adamietz A, Rahn R. Prophylaxis with povidoneiodine against induction of oral mucositis by radiochemotherapy. Support Care Cancer 1998; 6:373-77.

42. Sharma R, Tobin P. Management of chemotherapy-induced nausea, vomiting, oral mucositis and diarrhoea. Lancet Oncol 2005;6:93102.

43. Epstein JB, Tsang A. The role of salivary function in modulating chemotherapy-induced oropharyngeal mucositis: A review of the literatura. Oral Surg Oral Med Oral Pathol Oral Radiol Endod 2002;94: 39-44.

44. Plevova P. Prevention and treatment of chemotherapy-and radiotherapy- induced oral mucositis: a review. Oral Oncol 1999;35:453-70.

45. Migliorati CA, Siegel MA, Elting LS. Bisphosphonate-associated osteonecrosis: a long-term complication of bisphosphonate treatment. Lancet Oncol;7:508-14.

46. Mgliorati CA, Casiglia J, Epstein J, Managing the care of patients with bisphosphonate-associated osteonecrosis. J Am Dent Assoc 2005;136: 1658-68. 